\title{
A NOTE ON A SEPARATION OF EQUATIONS OF VARIATION OF THE ELLIPTIC RESTRICTED THREE-BODY PROBLEM INTO HILL'S EQUATIONS
}

\author{
V. MATAS \\ Astronomical Institute of the Czechoslovak Academy of Sciences, \\ Budečská 6, Vinohrady, Praha 2, Czechoslovakia
}

\begin{abstract}
The equations of variation of the three-dimensional elliptic restricted three-body problem corresponding to the equilibrium solutions (the libration points) have been separated into three Hill's equations. As regards the equation 'corresponding' to the motion of the infinitesemal body in the $z$-axis (perpendicular to the plane of motion of the primaries), the matter is trivial one since the initial equation - as known - reads $\mathrm{d}^{2} z / \mathrm{d} v^{2}+\left(A_{i}+e \cos v\right) /(1+e \cos v)=0(e, 0<e<1$, and $v$ are the eccentricity and the true anomaly of the relative motion of the primaries) with $A_{i}>1$ for the straight-line libration points $L_{i}(i=1,2,3)$ and $A_{i}=1$ for the triangular libration points $L_{i}, i=4,5$. As concerns the remaining two components, $x$ and $y$, of the motion of the infinitesimal body ( $x, y$ and $z$ are the Nechvile's variables), in the case of the straight-line libration points, $L_{1}, L_{2}$ and $L_{3}$, the corresponding equations of variation have been transformed and separated into two further - mutually independent - Hill's equations without any limitation. In the case of the equilateral triangle libration points, $L_{4}$ and $L_{5}$, the separation has been found only when the eccentricity $e$ and the dimensionless mass $\mu, 0<\mu \leqq \frac{1}{2}$, of the 'minor' primary satisfy the additional conditions:
\end{abstract}

$$
\begin{gathered}
\frac{1}{9}\left\{5+e^{2}-\left[8\left(e^{2}+2\right)\left(1-e^{2}\right)\right]^{1 / 2}\right\} \neq 3 \mu(1-\mu)< \\
<\frac{1}{9}\left\{5+e^{2}+\left[8\left(e^{2}+2\right)\left(1-e^{2}\right)\right]^{1 / 2}\right\} .
\end{gathered}
$$

Let us write the latter two Hill's equations obtained in the form

$$
\mathrm{d}^{2} y_{k}^{*} / \mathrm{d} v^{2}+I_{k}(v) y_{k}^{*}=0, \quad k=1,2,
$$

where $I_{k}, k=1,2$, are $2 \pi$-periodic even functions of the true anomaly $v$. The functions $I_{k}, k=1,2$, are real functions in the case of the straight-line libration points, $L_{1}, L_{2}$ and $L_{3}$, without a limitation but in the case of the triangular libration points, $L_{4}$ and $L_{5}$, they are real only if

$$
3 \mu(1-\mu)<\frac{1}{9}\left\{5+e^{2}-\left[8\left(e^{2}+2\right)\left(1-e^{2}\right)\right]^{1 / 2}\right\} .
$$

Provided

$$
\begin{aligned}
\frac{1}{9}\left\{5+e^{2}\right. & \left.-\left[8\left(e^{2}+2\right)\left(1-e^{2}\right)\right]^{1 / 2}\right\}<3 \mu(1-\mu)< \\
& <\frac{1}{9}\left\{5+e^{2}+\left[8\left(e^{2}+2\right)\left(1-e^{2}\right)\right]^{1 / 2}\right\}
\end{aligned}
$$

the functions $I_{k}, k=1,2$, are complex-valued functions of the real variable $v$. 